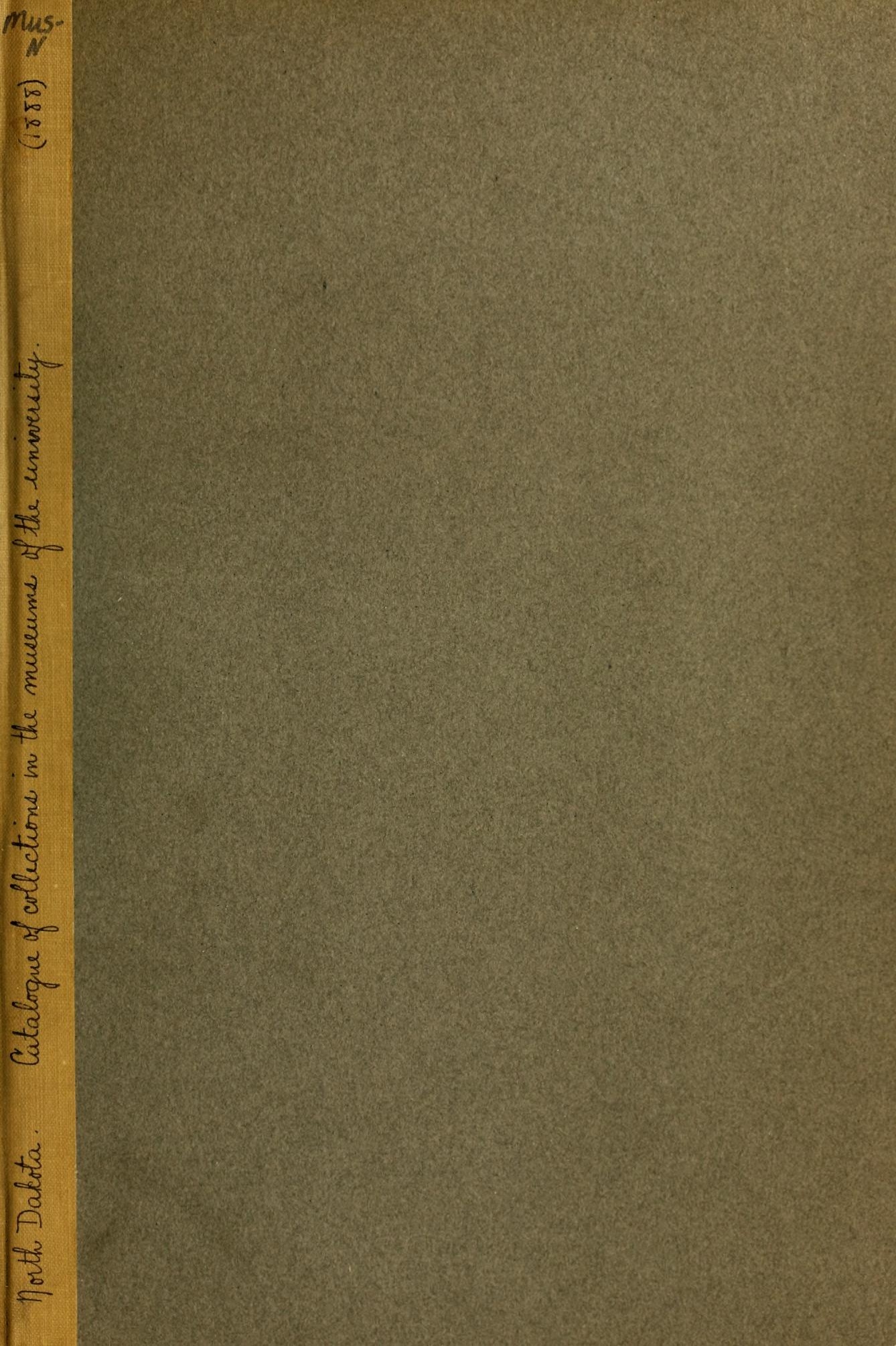




\section{HARVARD UNIVERSITY.}

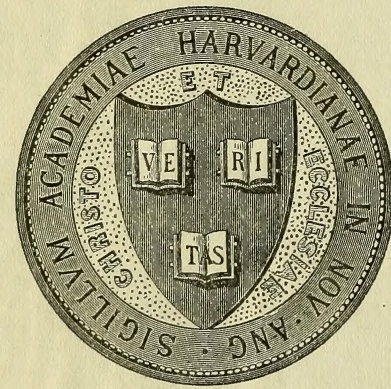

LIBRARY

OF THE

MUSEUM OF COMPARATIVE ZOÖLOGY 07,676

GIFT OF Harrard college library.

September 28, 1921 . 


\section{$5 \%, 676$}

$$
\begin{aligned}
& \text { CATALOGUE } \\
& \text { OF THE }
\end{aligned}
$$

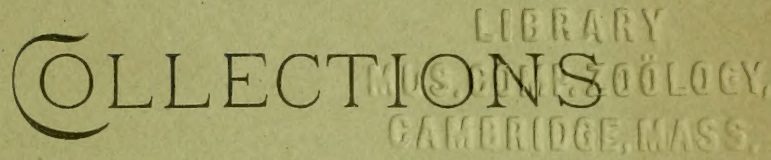

$$
\begin{aligned}
& \text { IN THE } \\
& \text { MUSEUMS } \\
& \text { OF THE } \\
& \text { UNIVERSITY } \\
& \text { OF } \\
& \text { NORTH DAKOTA. }
\end{aligned}
$$




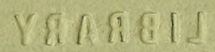

Monogesmos. cum

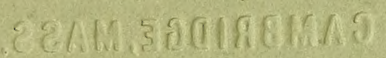




\section{CATALOGUE}

OF THE

\section{CLLECTIONS}

IN THE

MUSEUMSK

OF THE

UNIVERSITY

OF

NORTH DAKOTA.

PREPARED BY

HENRY MONTGOMERY, CURATOR.

GRAND FORKS, DAKOTA, 1888. 


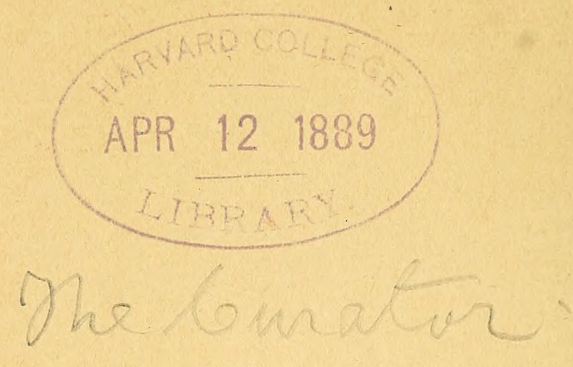

GRANI FORKS, DAKOTA:

PLAINDEALER BOOK AND JOB ROOMS. 1889. 


\section{BOARD OF REGENTS.}

His Excellency, the Governor of Dakota, Ex-Officio. Hon. W. N. Roach, ... ... ... Larimore.

Hon. F. R. Fulton, $\quad \ldots$... . ... Grand Forks Hon. James Twamley, ... . . . . . . Minto.

Hon. P. C. Donovan, M. I)., … ... Neche.

Hon. C. E. Heidel, A. M., … … Valley City.

President of the Board, ............. Won. W. N. Roach. President of the University, Homer B. Sprague, M. A., Ph. D. 


\section{MUSEUMS COMMITTEE \\ OF THE}

BOARD OF REGENTS.

IIon. P. C. Donovan, M. D.,............ Neche.

How. F. R. Fulton, ................... Grand Forks

Hon. C. E. Hendel, A. M., .............. Valley City.

CURATOR OF MUSEUMS,

Henry Montgomery, M. A., B. Sc., Ph. B.,..... Grand Forks 


\section{Jinerals, Fiocks and Fossils.}

\section{BLACK HILLS COLLECTION.}

Collected by H. Montgomery, in 1885, under the authority of the Board of Regents.

1 -Gold Ore. Native gold in porphyry. This weighs some 70 pounds and shows several nuggets on its surface.

Homestake mines, Lead City, Black Hills, Dakota.

2 3-Gold Ore. Gold ore in porphyry.

Homestake mines, Lead City. Black Hills, Dakota.

4-Gold Ore. Native gold in quartz rock.

Homestake mines, Lead City, Black Hills, Dakota.

5-7-Silver Ore. Cerargyrite or horn silver.

Carbonate Camp, Central City, Black Hills, Dakota.

8-11-Tin Ore. Cassiterite in Albite rock.

Etta mine, Harney Peak region, Black Hills, Dakota.

12-19-Tin Ore. Cassiterite in Greisen rock, quartzose rock, etc.

Harney Peak region, Black Hills, Dakota.

2021 -Tin Ore. Stream tin. Cassiterite.

Stream in Bear Gulch, west of Spearfish, Black Hills.

22-Tin Ore. Cassiterite in quartzose rock.

Bear Gulch, near Spearfish, Rawlinson's mining region, Black Hills, Dakota. 
23-Metallic Tin. Prepared from tin ore taken from the Etta mine.

Harney Peak region, Black Hills, Dakota.

24-Metallic Tin. Large bar prepared from "stream tin." Taken from the stream in Bear Gulch, at Nigger Hill, west of Spearfish, Black Hills, Dakota.

2526 -Copper Ore. Malachite or green carbonate of copper. Near Central City, Black Hills, Dakota.

27 -Copper Ore. Chalcopyrite or copper pyrites.

Black Hills, Dakota.

28--Lead Ore. Galena or sulphide of lead.

Black Hills, Dakota.

29-Lead and Silver Ore. Argentiferous galena.

Galena, south of Deadwood, Black Hills, Dakota.

30 31-Uranium Ore. Uran-mica.

Bald Mountain district, Black Hills, Dakota.

32-35-Zinc Ore.

Near Sturgis, Black Hills, Dakota.

36-Iron pyrites (iron disulphide).

Northwest of Spearfish.

37 38-Iron Ore. Hrmatite with mammillated surface.

Near Spearfish, Black Hills, Dakota.

39-Hæmatite with botryoidal surface.

Near Spearfish, Black Hills, Dakota.

40-Gypsum, massive and very pure.

Spearfish, Black Hills, Dakota.

41-Gypsum, with botryoidal surface.

Spearfish, Black Hills, Dakota.

42 43-Gypsum, crystallised Selenite, white and pearly.

Near Deadwood, Black Hills, Dakota.

44-Gypsum, crystallised Selenite, reddish.

Near Deadwood, Black Hills, Dakota. 
45-Plaster. Prepareal from gyjpsum of the Speartish district.

Spearfish, Black IIills, Dakota.

46-Ornament. Made from the Spearfish gypsum hy Mr. Peter 'Thane.

Speartish, Black Hills, Dakoia.

+7-(xypsum, massive.

Near Custer City, Black Hills, Dakota.

48-Gypsum, massive.

Southwest of Custer City, Black Hills, Dakota.

4!-52-Marble, variegated.

Gilman's quarries, near Sturesis and Boulder Park, Black Hills, Dakota.

53-Marble, variegated. Polished.

Gilman's quarries, near Sturgis, Black Hills, Dakota.

5 t-Marble, variegated, polished.

Gilman's quarries, near Stmrgis, Black Hills, Dakota.

55-Marble, variegated, partially polished.

Near Sturgis, Black Ilills, Dakota.

56-Marble, white, polished.

Black Hills, Dakota.

57-66-Coal. Lignite.

Near Hay Creek, northwest of Speartish, Black Hills, Dakota.

67 68-Grey building stone. Compact, silicenus limestone.

Black Ilills, Dakota.

69-Red building stone.

Black IIills, Dakotar.

70-81-Miea, of various colers; thin, hroad plates. Some of these are quite transparent, and suitalse for use in stoves and lanterns.

('uster ristrict, Jiack Mills, D)akota.

82 S3-Mica.

Ingersoll mine, Iarney Peak District, Jilack Hills.

Dakota. 
84-Garnet crystals, large, imbedded in slate.

Harney Peak district, Black Hills, Dakota.

S5-Garnet Crystals, smaller, imbedded in slate.

Pine Camp, Harney Peak region, Black Hills, Dakota.

86-Garnet crystals, small and numerous.

Taken from sands in the bed of stream in Harney Peak region, Black Hills, Dakota.

87-Tourmaline crystal, black. Large specimen.

Harney Peak region, Black Hills, Dakota.

88-Tourmaline crystal, black. Large specimen.

Hai ey Peak region, Black Hills, Dakota.

\&9 90-Black Tourmaline crystals in Mica.

Harney Peak region, Black Hills, Dakota.

91-95-Green Tourmaline erystals in Mica.

Ingersoll mine, Harney Peak region, Black Hills, Dakota.

96 97-Black 'Tourmaline acicular erystals, in fine gangue.

Harney Peak region, Black Hills, Dakota.

98--Larger black Tourmaline crystals in tine gangue.

Harney Peak region, Black Hills, Dakota.

99-Tourmaline crystals in white quartz.

Black Hills, Dakota.

100101 -Tourmaline crystals in Greisen rock.

Black Hilis, Dakota.

102 103--Rose Quartz.

104--Milky Quartz.

Black Hills, Dakota.

Black Hills, Dakota.

105-Rock crystal. Transparent.

Black Hills, Dakota.

106-Drusy Quartz. Aggregated crystals.

These are peculiar, spongy and coralloid clusters.

Wind Cave, South of Deadwood, Black Hills, Dakota.

107-109-Drusy calcite, Aggregated crystals.

Wind Cave, Black Hills, Dakota. 
110-Chalcedony, in chain-like masses, and shading into Onyx.

Black Hills, Dakota.

111-Chalcedony.

Black Hills, Dakota.

112-Agate.

Black Hills, Dakota.

113-Banded Agate.

Black Hills, Dakota,

114-Jaspery Quartz.

Black Hills, Daknta.

115-119-Spodumene (Lithia, Silica and Alumina), large crystals. Harney Peak region, Black Hills, Dakota.

120-Spodumene, carrying Vanadinite.

Harney Peak region, Black Hills, Dakota.

121 122-Beryl Crystals. Large hexagonal prisms, each face four inches wide and two feet long.

Harney Peak region, Black Hills, Uakota.

123-Beryl Crystal. Green.

Harney Peak region, Black Hills, Dakota.

124-Granite.

Black Hills, Dakota.

125 126-Graphic Granite.

Black Hills, Dakota

127-Porphyry.

Deadwood, Black Hills, Dakota.

128-Dendrite.

Lead City, Black Hills, Dakota.

129-Dendrite on Rhyolite.

Lead City, Black Hills, Dakota.

130-132--Conglomerate.

Lead City, Black Hills, Dakota.

133-Breccia. Unusually fine.

Hot Springs, Black Hills, Dakota. 
134-Homblemble Rock, with imbedded erystais of Iron pyrites.

135 -dsbestos.

Black Hills, Dakota.

Black Hills, Dakota.

1:36-Dog-tooth Spar. Variety of Calcite. Large slab of crystals, yellowish in color.

Black Hills, Dakota.

137-Dog-tooth Spar. Variety of Calcite, aggregation somewhat different from the preceding... White and passing to the glassy and almost colorless.

Black Hills, Dakota.

1::8-Dogtooth Spar. Aggregation of crystals, differing from the foregoing. Color buff to reddish.

Spring Creek, Black Hills,--Dakota.

139-Nail-headed Spar. Variety of Calcite. Aggregation of "nail-headed" crystals. Yellowish in color.

Black Hills, Dakota.

140-Nail-headed Spar. Aggregation of "nail-headed" erystals. White in color.

Black Hills, Dakota.

141-152-Calcite crystals. Large crystals from the roof and walls of Whitewood Cave, six miles northeast of Deadwoor.

Black Hills, Dakota.

15:-155-Rerl and White: Lime Rock, showing concentric rings of color.

Lead City, Black Hills, Dakota.

156-158-Columbite: Crystals. Large and fine.

Harney Peak region, Black Hills, Dakota.

159-Banded Sandstone.

Near Deadwood, Dakota.

160-Geode and vug.

Black Hills, Dakota.

161-Utica Shale, with Trilobite impressions.

Custer City, Black Hills, Dakota.

102-Concretion.

Black Hills, Dakota. 
163-Petrified Wood.

Black Hills, Dakota.

164--Petrified Wood.

Black Hills, Dakota.

165-Petrified Wood. Near Mato 'Tipi (Bear's Lodge or Devil's Tower). Mato Tipi is a rock in the northwestern part of the Black Hills. It is alnost cylindrical and perpendicular, consists of volcanic trachyte, is 376 feet in diameter at its summit, and is 625 feet in height.

166 167-Crude Petroleum in bottles.

From well near Mato Tipi.

168-Ore of Graphite or Plumbago.

Black Hills, Dakota.

169 170-Rock, carrying fossil leaves.

"Bad Lands," near the Black Ilills, Dakota.

171-174--Fossiliferous Limestone Rock. These specimens eontain many small shells, most of which are bivalve Entomostraca, and so far as known to the author, their first discovery in Dakota was made by bim in August, 1885.

Near Spearfish, Black Hills, Dakota.

175-Large slab of rock, carrying fossil biválve shells.

Buffalo Gaj, Black Hills, Dakota.

176-Fossil Inoceramus. A large bivalve shell beloniging to the Lamellibranchiate molluscs.

From "Bad Lands," near the Black Hills, Dakota.

177-Fossil bivalve shell.

From "Bad Lands," near the Black Ilills, Dakota.

178-Mass of fossil Gasteropod and other shells, cemented together by lime.

From "Bad Lands," near the Black Hills, Dakota. 


\section{CLASS CEPHALOPODA.}

ORDER TETRABRANCHIATA-FAMILY AMMONITIDAE.

179--Ammonites placenta. Very large fossil shell, one foot in diameter, showing branched and complex septa.

"Bad Lands," near Black Hills, Dakota.

180-Baculites sp.? Baculite. With pearly shell, well preserved.

"Bad Lands," near Black Hills, Dakota.

181-Baculites sp.? Baculite. Shell wanting, septa very distinct.

"Bad Lands," near Black IIills, Dakota.

182-Scaphites nodosus. Shell well preserved.

"Bad Lands," near Black Hills, Dakota.

183-Scaphites nodosus, with Baculites.

"Bad Lands," near Black Hills, Dakota.

184 185-Nautilus De Kayi. Showing pearly shell and the siphuncle.

"Bad Lands," near Black Hills, Dakota.

186-Fossil Turtle. Large specimen, showing plates and sutures of carapace and plastron. Colored reddish from presence of Iron Oxide.

"Bad Lands," near Rapid City, Black Hills, Dakota.

187--Skull of fossil carnivore, showing large canines and other teeth.

"Bad Lands," near Black Hills, Dakota.

The foregoing is but a portion of the collection made by the writer in the summer of 1885. Before it was damaged and scattered by the tornado of 1887 , this collection numbered 767 specimens. 
University of North Daliota. 


\section{1.-GENERAL GEOLOGICAL COLLECTION.}

Stratigraphical Series-1 to 101.

1-Granitoid Gneiss. Laurentian Period.

Westchester Co., N. Y:

2-Apatite Rock. Laurentian Period.

Burgess, Ontario.

3-Magnetic Iron Ore: Laurentian Period.

Port Henry, N. Y.

4--Hornblende Schist. Laurentian Period.

Antwerp, N. Y.

5-Hypersthenite. Laurentian Period.

Port Kent, N. Y.

6-Eozoon Limestone. Laurentian Period.

Thurman, N. Y.

7-Granite.

8-Contorted Gneiss.

9-Argillite. Huronian Period.

Thomson, Minn.

10-Granite. Huronian Period.

Clark's Island, Maine.

11-Syenitic Granite. Huronian Period.

Spruce Head, Maine.

12-Cambrian Slate. Cambrian Period.

Charnwood Forest, Wales.

13-Shale. Cambrian Period.

Braintree, Mass.

14-Potsdam Sandstone. Potsdam Period.

Potsdam, N. Y.

15-Potsdam Sandstone, with Lingula prima. Potsdam Period.

Keeseville, N. Y, 
16-Potsdam Sandstone. Potsdam Period.

Hinckley, Minn.

17-Magnesian Limestone. Canadian Period.

Near Madison, Wis.

18-Landeilo Flags: Lower Silurian.

Moffat, Scotland.

19-Calciferous Sandrock. Canadian Period.

Little Falls, N. Y.

20-Chazy Limestone. Trenton Period.

Chazy, N. Y.

$21-\quad$ Trenton Period. Nashville Group.

Nashville, Tenn.

22-Trenton Limestone. Trenton Period.

Trenton Falls, N. Y.

2:3- Cincinnati Group. Trenton Period. Cincinnati, Ohio.

24-Utica Slate. 'Trenton Period.

Utica, N. Y.

25-Hudson River Shale. Trenton Period.

Cohoes, N. Y.

26-Oneida Conglomerate. Medina Period.

Oneida Co., N. Y.

27-Medina Sandstone. Medina Period.

Medina, N. Y.

28-Medina Sandstone, with Lingula cuneata. Medina Period.

Medina, N. Y.

29-Clinton Iron Ore. Niagara Period.

Rochester, N. Y.

30-Pentamerus Limestone. Niagara Period.

Rochester, N. Y.

31--Cpuer Green Shale, with Graptolites. Niacara Period. 
32--Upper Green Shale, with Atrypa. Niagara Period. Rochester, N. Y.

33-Niagara Shale. Niagara Period.

Rochester, N. Y.

34-Encrinal Limestone. Niagara Period.

Rochester, N. Y.

35-Niagara Limestone, Niagara Period.

Niagara Falls, N. Y.

36-Gypsiferous Shale. Salina Period.

Jamesville, N. Y.

$37 \ldots$ Waterlime Group, Helderberg Period.

Buffalo, N. Y.

38-Tentaculite Limestone. . Helderberg Period,

Schoharie, N. Y.

39-Lower Pentamerus Limestone. Helderberg Period.

Schoharie, N. Y.

40--Oriskany Sandstone. Oriskany Period, Devonian.

Oriskany Falls, N. Y.

41--Cauda-Galli Grit. Corniferous Period.

Rondout, N. Y.

42-Cherty Limestone. Corniferous period.

Buffalo, N. Y.

43-Marcellus Shale. Hamilton Perind.

Le Roy, N. Y.

44-Encrinal Limestone. Hamilton Period.

Erie Co., N. Y.

45-Moscow Shale, Hamilton Period.

Moscow, N. Y.

46-Genesee Slate, Hamilton Period

Moscow, N. Y.

47-Gardean Shale, Chemung Poriod.

Livingstone Co., N. Y. 
48-Portage Sandstone. Chemung Period.

Portageville, N. Y.

$49-$ Chemung Group. Chemung Period Elmira, N. Y.

50-Chemung Rock, with Dictyophyton. Chemung Period, Devonian.

Steuben Co., N. Y.

5 1-Panama Conglomerate. Chemung Period.

Panama, N. Y.

52-Panama Conglomerate. Chemung Period.

Panama, N. Y.

$53-$

Catskill Period, Devonian.

Delaware Co., N. Y."

54-Old Red Sandstone. Catskill Period.

Dura Den, Scotland.

55-Devonian Limestone. Catskill Period.

Torquay, England.

56-Oolitic Limestıne. Sub-Carboniferous Period.

Bristol, England.

57- Waverly Sandstone. Sub-Carboniferous Period.

Berlin, Ohio.

5s-Burlington Limestone. Sub-Carboniferous Period.

Burlington, Iowa.

59-St. Louis Limestone. Sub-Carboniferous Period.

St. Louis, Mo.

60-Carboniferous Conglomerate. Carboniferous Period.

Hampden, Ohio.

61-Millstone Grit. Carboniferous Period.

Yorkshire, England.

62-Millstone Grit. Carboniferous Period.

Manebach, Thuringia.

63-Coal Shale. Carboniferous Period. 
64-Coal Shale. Carboniferous Period.

Pittston, Penn.

65-Anthracite. Carboniferous Period.

Lehigh Valley, Penn.

66-Cannel Coal. Carboniferous Period.

Grayson, Ky.

67-Oolitic Limestone. Carboniferous Period.

Kansas City, Mo.

68- Permian Period.

Trebnitz, Saxony.

69-Portland Sandstone. Triassic Period.

Portland, Conn.

70-Limestone. Triassic Period.

South Britain, Conn.

71-Bunter Sandstein. Triassic Period.

Val d'Ayol, France.

72-New Red Sandstone. Triassic Period.

Worcestershire, England.

73-Muschelkalk. Triassic Period.

Brunswick, Germany.

74-Keuper Sandstone.

Uttoxeter, England.

76-Middle Lias Shale.

Lyme Regis, England.

77--Upper Lias Shale. Jurassic Period.

Near Boll, Wurtemberg.

78--Upper Lias Limestone. Jurassic Period.

Calvados, France.

79 Lower Oolite. Jurassic Period.

Bridgeport, England. 
su-Bath Oolite. Jurassic Period.

$$
\text { Bath, England }
$$

81-Lithographic Limestone. Jurassic Period.

Solenhofen, Bavaria.

82-Limestone Niddle Oolite. Jurassic Period.

Saskale, Karpathen.

83-Portland Oolite. Jurassic Period.

Portland, England.

84--Limestone. Jurassic Period.

Mt. of Olives, Palestine.

85-Greensand. Cretaceous Period.

Folkestone, England.

86- Cretaceous Period. Dakota Group.

Ottawa Co., Kansas.

87-"Greensand." Cretaceous Period.

Marlborough, N. J.

88--White Chalk. Cretaceous Period.

Dover, England.

89-Flint Nodule. Upper Chalk. Cretaceous Period.

Dover, England.

90-Nummulitic Limestone. Tertiary Period.

Near Cairo, Egypt.

91- Bridger Group. Eocene. Tertiary Period.

Smith's Fork, Wyoming.

92-Variegated Sand. Tertiary Period.

Alum Bay, Isle of Wight.

93-Calcaire Grossier. Tertiary Period.

Arcueil, France.

94-_ Tertiary Period.

Mt. Lebanon, Asia Minor.

95-Diatomaceous Earth. 'T'ertiary Period.

Richmond, $\mathrm{V}$, 
96--Basalt. Tertiary Period.

Giant's Causeway, Ireland.

97--Coralline Crag. Pliocene. Tertiary Period.

Oiford, England.

98--Puddingstone (Diluvium). Quaternary Period.

Poughkeepsie, N. Y.

99-Champlain Clay. Quaternary Period.

Albany, N. Y.

100--Shell Marl, containing fresh-water shells. Quaternary Period.

Monroe Co., N. Y.

101-Calc Tufa. Quaternary Period.

Delphi, N. Y.

102-Striated Limestone. Large slab showing ancient glacial strix.

Rochester, N. Y.

103--Glaciated Boulder.

Rochester, N. Y.

104-Sun Cracks and Mud Veins.

Missouri.

105-Septarium.

Kansas.

106-Rock triturated by glaciers.

Mer de Glace, Switzerland.

107-Sigillaria. Fossil plant, extinct. Portion of a large tree trunk.

Carboniferous Rocks, Penn.

108-Lepidodendron. Fossil and extinct plant. Portion of a tree trunk.

Carboniferous Rocks, Indiana.

109-113-Specimens of Gold and Silver Ore. From near Juneau, Alaska.

Presented by the Hon. M. L. McCormack, Grand Forks, Dakota, 1888. 
114-116-Halite or Rock Salt, in lump. Taken 750 feet beneath the surfice. Piffard, Livingstone Co., N. Y.

Presented by O. M. Hopkins, Esq., Grand Forks.

117-Halite or Rock Salt, refined. Piflard, Livingstone (o., N. Y.

Presented by O. M. Hopkins, Esq., Grand Forks.

118-IIalite or Rock Salt, in lump.

Presented by G. B. Winship, Esq., Grand Forks.

119-121-Polished Granite of different kinds. Maine, U. S.

Presented by C. P. Trepanier, Esq., Grand Forks.

122 123-Mottled Marble, polished. Swanton quarries, Vt.

Presented by E. Ranson, Esq., Grand Forks.

124-Encrinital Marble, polished. Swanton quarries, Vt.

Presented by E. Ranson, Esq., Grand Forks.

125126 -Catlinite or Pipestone. Pipestone City, Minn.

Presented by Geo. Harvey, Esq., Minto, Dakota.

127-Group of Rock Crystals.

Rocky Mountains, Canada.

128-Tin Ore. "Stream Tin." Ferruginous and Siliceous Cassiterite. Near Helena, Montana.

Presented by W. H. Hackney, Esq., Minneapolis.

129-Rock. Granitoid. From Mer de Glace, Chamonix, Savoy.

Presented by Prof. John Macnie, University of North Dakota, October, 1888.

130-Sand of the White Sand Rock Formation. Taken, at a depth of 1500 feet, from an artesian well in Devils Lake City, Ramsey Co., Dakota, Nov. 1888.

Presented by Mayor H. C. Hansbrough of Devils Lake, Dakota.

131-Fluorspar. Purple crystals (cubes).

Cumberland, England.

132-Steatite or Soapstone.

Georgia.

133-Obsidian. Siliceous and volcanic.

Lipari Isles, Mediterranean. 
134-Pumice. Variety of obsidian.

Lipari Isles, Mediterranean.

135-Cellular Basalt.

Lipari Isles, Mediterranean.

136-Fossiliferous rock, containing shells of Brachiopods. Near Missouri River.

Presented by Regent Twamley, in 1885 .

137-Banded calcareous rock, or marble. Gibraltar, Spain. Presented by Mrs. C. L. Wording, Grand Forks, '86.

138-Amethyst crystals. North Shore of Lake Superior, Canada.

Presented by Henry Montgomery, University of North Dakota.

139-Specular Iron Ore. Near Marquette, Michigan.

Collected and presented by Henry Montgomery, University of North Dakota.

140-142-Champlain Clay. Quaternary Period. Taken by the Government dredge (in charge of $\mathrm{Mr}$. Davenport) several feet below the bed of the Red River, near Grand Forks, in 1887.

Samples presented by Henry Montgomery, University of North Dakota.

143-Cedar wood. Taken from a tree, ten inches in diameter, found, in 1883, in a horizontal position, nine feet beneath the bottom of a coulee, on the farm of Mr. Alex. McKenzie, Milan Township, Grand Forks Co., Dakota.

Presented by Alex. McKenzie, Esq., in 1886.

144-Selenite. A ball of twin crystals found in clay many feet below the surface, near the Turtle Mountains, Dakota.

Presented by Mr. G. Makee, of Dunseith, in 1888.

145-Peat or Tarf.

Haspelmoor, Bavaria.

146-Serpentine.

Bridgeport, Conn. 
University of North Dalkota.

23

147. Homalite Red inon Oxide.

Near Hower, Himuesot.

Resented dy_Regent'C. E. Reideb, 1889 


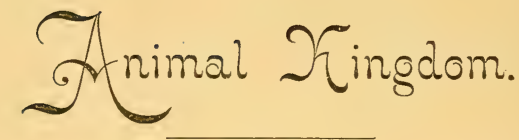

The marine invertebrates received from the Smithsonian Institution are indicated by the initials S. I. Most of them are wet specimens.

SUB-KINGDOM-SPONGIDA.

1-Euplectella aspergillum. Venus's Flower Basket. Skeleton of a siliceous sponge consisting of interlacing glassy fibres.

Philippine Islands.

2-Hyalonema Sieboldii. Glass Rope Sponge.

Japan.

3-Spongia tubulifera. Tube sponge. Skeleton of sponge consisting of horny fibres.

West Indies.

4-Verongia fistularis. Cup Sponge. Skeleton.

West Indies.

5--Cliona sulphurea (Verrill, Yale University). Boring Sponge. S. I.

Vineyard Sound, Mass.

\section{SUB-KINGDOM-CCELENTERATA.}

CLASS-ACTINOZOA.

6--Cerianthus Lloydii. Sea-Anemnne. Glass model.

7-Hyanthus Mitchelli. Sea-Anemone. Glass model.

8-Meandrina labyrinthica. Brain Coral. Dried skeleton.

West Indies. 
9-Meandrina confluentes. Brain Coral. Dried skeleton.

West Indies.

10-Fungia echinata. Mushroom Coral. Skeleton.

Java.

11-Dendrophyllia nigrescens. Black Tree Coral. Skeleton.

Tonga Isles.

12-Madrepora secunda. Madrepore Coral.

West Indies.

13--Tubipora musica. Organ-pipe Coral.

14-Eugorgia aurantiaca. Yellow Sea-fan.

Bahamas.

15-Pennatula aculeata. Sea Feather. S. I.

Off Martha's Vineyard, Mass.; 207 fathoms.

16-Acanella Normani. Jointed Bush Coral. S. I.

Off Martha's Vineyard, Mass.; 200 fathoms.

17-Metridium marginatum. Common Sea Anemone. S. I. Newport, Rhode Island, shore.

18-Urticina nodosa. Warty Sea Rose. S. I.

Off Martha's Vineyard, Mass.; 191 to 245 fathoms.

19-Sagartia abyssicola. Deep Water Sea Rose. S. I.

Off Martha's Vineyard, Mass.; $16+$ to 229 fathoms.

20-Epizoanthus Americanus. S. I.

Off Martha's Vineyard, Mass.; 19:3 fathoms.

CLASS-IIYDROIDEA.

21-Obelia geniculata (Hincks, Toronto University). S. I. Off Martha's Vineyard, Mass.; 97 fathoms. 22-Sertularella polyzonias (Gray). S. I.

Off Cape Cod, Mass.; 27 fathoms. 
2:3-Sertularella tricuspidata (Hincks). S. I.

Off Cape Cod, Mass.; 18 fathoms.

24-Globiceps tiarella (Ayres). S. I.

Buzzard's Lay, Mass.

SUB-KINGDOM-ECHINODERMATA.

ORDER- CRINOIDEA.

25-Antedon dentatum. Feather Star. S. I.

Off Martha's Vineyard, Mass.; 146 to 183 fathoms. ORDER-OPHIUROIDEA:

26-Ophiopholis aculeata. Variegated Serpent Star. S. I. Off Martha's Vineyard, Mass.; 146 fathoms. 27-Amphiura macilenta. S. I.

Off Martha's Vineyard, Mass.; 63 fathoms. 28-Ophiocantha millespina. S. I.

Off Martha's Vineyard, Mass.; 234 fathoms.

29 30-Ophioglypha Sarsii. Sars' Serpent Star. S. I.

Off Martha's Vineyard, Mass.; 46 to 100 fathoms.

31-Astrophyton Agassizii. Basket Fish. S. I.

Off Cape Cod, Mass.

ORDER-ASTEROIDEA.

32-Asterias ochracea. Ochre-colored Star-fish.

California coast.

33 34-Solaster papposus. Sun Star.

Heligoland, German Ocean.

35-Asterias Forbesii. Forbes' Star-fish.

36-Asterias Forbesii. Common Star-fish or Forbes' Starfish. S. I.

Vineyard Sound, Mass. 
37-Leptasterias compta. S. I.

Off Newport, R. 1.; 27 fathoms.

38-Ctenodiscus crispatus. Velvet Star. S. I.

Massachusetts Bay.

39-Archaster Americanus. S. I.

Off Martha's Vineyard, Mass.; 89 to 202 fathoms.

ORIER-ECHINOIDEA.

4041 -Echinus elegans. Sea-urchin.

German Ocean.

42-Heterocentrotus trigonarius. With thick spines from one to four inches in length.

Mauritius.

4344 -Sphærechinus granularis.

Mediterranean Sea.

4.)-Strongylocentrotus drobachiensis. Common Sea-urchin or Sea-egg. S. I.

Off Newport, Rhode Island.

46-Arbacia punctulata. Purple Sea-urchin. S. I.

Vineyard Sound, Mass.

47 48-Echinarachnius parma. Sand Dollar. S. I.

Off Martha's Vineyard, Mass.; 26 fathoms.

ORDER-HOLOTHUROIDEA.

49-Holothuria atra. Black Sea-cucumber or Trepang. In alcohol.

Samoa.

50-Thyone Briareus. Sea-encumber. S. I.

Wood's Holl, Mass., shore.

51-Leptosynapta Girardii. S. I.

Wnod's Holl, Mass, shore. 


\section{SUB-KINGDOM-VERMES.}

ORDER-CESTODEA.

52--Cysticercus cellulosæ. Immature and encysted stage of the common tape worm.

$$
\text { ORDER-CH ETOPODA. }
$$

53--Spirographis Spalanzanii.

Adria.

54--Lepidonotus squamatus. S. I.

Buzzard's Bay, Mass.

55--Lætmatonice armata. S. I.

Off Martha's Vineyard, Mass.; 225 to 319 fathoms.

56 57-Hyalinœcia artifex. Tubes or shells only. S. I.

Off Martha's Vineyard, Mass.; 250 fathoms.

58-Euglycera dibranchiata. S. I.

Southern New England Shore.

ј9-Chætopterus pergamentaceus. S. I.

Wood's Holl, Mass., shore.

60--Trophonia affinis. S. I.

Narragansett Bay, R. I., 10 to 20 fathoms.

61-Cirratulus grandis. S. I.

Southern New England, shore.

62-Spirorbis borealis. S. I.

Wood's Holl, Mass.

ORDER-GEPHYREA.

63-Phascolosoma Gouldii. Sipunculoid Worm. S. I. Wood's Holl, Mass., shore.

64-Phascolion Strombi. Sipunculoid Worm. S. I. Off Newport, R, I, 12 to 19 fathoms, 
ORHER-CHLATOGNATHA.

65--Sagitta elegans. S. I.

Vineyard Sound, Mass., surface.

ORDER-POLYYOA.

66-Cellularia ternata. S. I.

Otf Cape Cod, Mass.; 18 fathoms.

67-Gemellaria loricata. S. I.

Oft Cape Cod, Mass.; 24 to.30 fathoms.

68-Bugula turrita. S. I.

Narragansett Bay, R. I.; 4 to (; fathoms. 69-Electra pilosa. S. I.

Southern coast of New England.

ORDER-TUNICATA.

70-Molgula manhattensis. S. I.

Southern New England, shore.

71-Halocynthia partita. S. I.

Wood's Holl, Mass., shore.

72-Botryllus Gouldii. S. I.

Wood's Holl, Mass.

73-Perophora viridis. S. I.

Wood's Holl, Mass.

74-Amorœcium pellucidum. S.I.

Vineyard Sound, Mass.

75-Amornecium constellatum. S. I.

Vineyard Sound, Mass.

76-Amorcecium stellatum. S. I.

Wood's Holl, Mass.

77-Leptoclinum albidum. S. I.

Vineyard Sound, Mass. 
78--Salpa caboti. S. I.

Vineyard Sound, Mass., surface.

79-Salpa (large species). S. I.

Off Martha's Vineyard, Mass., surface.

SUB-KINGDOMI-MOLLUSCA-(Shell-fishes.)

CLASS-LAMELLIBRANCHIATA.

80-Ensatella Americana. Razor Shell. S. I.

Long Island Sound, shore.

81-Mya arenaria. Long Clam. S. I.

Wood's Holl, Mass., shore.

82-Clidiophora trilineata. S. I.

Buzzard's Bay, Mass.

83-Callista convexa. S. I.

Narragansett Bay, R. I.

84-Tottenia gemma. S. I.

Provincetown, Mass., shore.

85-Cyprina islandica. S. I.

Off Newport, R. I.

86-Nucula proxima. S. I.

Southern coast of New England.

87-Scapharca transversa. S. I.

Buzzard's Bay, Mass.

88-Mytilus edulis. Common Mussel. S. I.

Buzzard's Bay, Mass.

89-Modiola plicatula. Ribbed Mussel. S. I.

Wood's Holl, Mass., shore.

90-Pecten irradians. Common Scallop. S. I.

Southern Coast of New England.

91-Pecten tenuicostatus. Smooth Scallop. S. I.

Off Martha's Vineyard, Mass.; 46 fathoms. 
92-Pecten tenuicostatus. Smooth Scallop. S. I.

Off Martha's Vineyard, Mass.

93-Anomia glabra. Silver-shell, Gold-shell or Jingleshell. S. I.

Southern coast of New England.

9495-Lithodomus lithophaga.

Mediterranean Sea.

96 97-Hippopus maculatus. Bear's Paw Clam.

Indian Ocean.

98 99-Solen siliqua. Large Razor Shells.

England.

100-103-Unio sp.? In alcohol.

Red River, Grand Forks, Dakota.

104-107-Anodon sp.? In alcohol.

Red River, Grand Forks, Dakota.

CLASS-GASTEROPODA.

108-Helix concava. Land Snail. Toronto, Ontario.

Presented by Henry Montgomery.

109-112-Paludina decisa. River Snail.

Red River, Grand Forks, Dakota.

113-115-Murex regius.

Panama, Cențral America.

116-Murex inflatus. 
118-121-Strombus granulosus.

Panama.

122--Strombus lentiginosus.

West Indies.

123--Pteroceras lambis. Wing Shell.

China.

124-Cassis rufa.

Brazilian coast.

125-127-Fusus sp.?

128-Fusus colus. Spindle Shell.

Ceylon.

129-Harpa ventricosa. Harp Shell.

Mauritius.

130--Potamides sp.?

131--Conus sp.? Cone Shell.

132-136--Trochus obeliscus. Top Shell.

137-Turbo sp.?

138 139-Nerita ustulata.

140-145--Natica sp.?

146-Oliva sp.? Olive Shell.

147 148-Cypræa sp.? Cowry Shell.

149 150-Cypræa sp.? Cowry Shell. 
151-Bulla ampulla. Bubble_Shell.

India.

152-Patella sp.? Sea Limpet.

153-156-Busycon perversum (?).

Florida.

157-Buceinum sp.? Whelk Shell.

15s-Buccinum undatum. Whelk. S. I.

New England coast.

159-Tritia trivittata. S. I.

Buzzard's Bay, Mass.

I60-Ilyanassa obsoleta. Black Mud-snail. S. I.

Newport, R: I., shore.

161-Urosalpinx cinera. S. I.

Wood's Holl, Mass., shore.

162-Purpura lapillus. Purple Sea-snail. S. I.

Wood's Holl, Mass., shore.

163-Lunatia heros. Sea-snail. S. I.

Vineyard Sound, Mass.

164-Littorina littorea. Periwinkle. S. I.

Wood's Holl, Mass., shore.

165-Littorina palliata. S. I.

Wood's Holl, Mass., shore.

166-Littorina rudis, S. I.

Wood's Holl, Mass., shore.

167-Bittium nigrum. S. I.

Wood's Holl, Mass., shore. 168-Crepidula fornicata. Boat Shell or Donble Decker. S. I. Buzzard's Bay, Mass. 
169-Acmœa testudinalis. Limpet. S. I.

Eastport, Maine, shore.

170-Melampus lineatus. Salt Marsh Snail. S. I.

Newport, R. 1., shore.

171-Pleurobranchia tarda. S. I.

Off Martha's Vineyard, Mass.; 100 to 124 fathoms.

CLASS-CEPHALOPODA.

172_Nautilus pompilius. Pearly Nautilus. Shell.

173-Argonauta argo. Paper nautilus. Shell.

Messina, Sicily.

174-Spirula peronii. Internal shell.

San Domingo, Wést Indies.

175-Octopus vulgaris. Octopus (cattle-fish). In alcohol.

Gulf of Mexico.

176--Loligo Pealei. Squid. S. I.

Southern coast of New England.

177--Loligo Pealei. Squid. Eggs. S. I.

Southern coast of New England.

\section{SUB-KINGDOM-ARTHROPODA.}

\section{Class-Crustacea.}

ORDER-CIRTIPEDIA.

178-Coronula balænaris. Animal in alcohol.

Tonga Isles.

179-Coronula balænaris, in whale's skin.

180-Coronula balænaris. Shell. 
181-'Tetraclita porosa. Acorn Shells, on rock.

Aden, Arabia. 182-186-Lepas anatifera. Barmacles in alcohol.

Indian Ocean.

187-Lepas anatifera. Barnacle. S. I.

Off Martha's Vineyard, Mass.

185--Balanus balanoides. Rock Barnacle or Acorn Shell. S. I.

New Haven, Conn., shore.

ORDER-ENTOMOSTRACA.

189-Artemia gracilis. Brine Shrimp. S. I.

New Haven, Conn.

ORDER-ISOYODA.

190-Cirolana concharum. S. I.

Wood's Holl, Mass.

ORDER-ANPHIPODA.

191-Orchestia agilis. Sand Flea or Beach Flea. S. I.

Newport, R. I., shore.

192-Talorchestia longicornis. Large Sand Flea. S. I.

New Haven, Conn., shore.

ORDER-SCHIZOPODA.

193-Mysis mixta. S. I.

Cape Cod Bay, Mass.; 27 fathoms.

ORDER-MEROSTOMATA.

194-Limulus polyphemus. King Crab or Horse-shoe Crab. Massachusetts.

ORIER-IIECAP(I)A.

195-Gelasimus pugnax. Fiddler Crab. S. I.

Wood's Holl, Mass., shore.

196-Gelasimus pugilator. Fiddler Crab. S. I.

Wood's Hnll, Mass., shore. 
197-Plationichus ocellatus. Lady Crab. S. I.

Vineyard Sound, Mass.

198-Cancer irroratus. Rock Crab. S. I.

Vineyard Sound, Mass.

199-Hyas coaretatus. S. I.

Coast of New England; 45 fathoms.

200-Libinia emarginata. Spider Crab. S. I.

Vineyard Sound, Mass.

201-Zoeas and Megalops of Crabs. S. I.

Vineyard Sound, Mass., surface.

202-Euprognatha rastellifera. S. I.

Off Martha's Vineyard, Mass.; 67 to 79 fathoms.

203-Hippa talpoida. Sand-brg or Bait-bug. S. I.

Wood's Holl, Mass., shore.

204-Eupagurus pollicaris. Hermit Crab. S. I.

New Haven, Conn.

205-Eupagurus longicarpus. Hermit Crab. S. I.

Narragansett Bay, 6 to 12 fathoms.

206-Eupagurus bernhardus. Hermit Crab. S. I.

Cape Cod Bay, Mass.; 15 fathoms.

207-Eupagurus politus. Deep-sea Hermit Crab. S. I. Off Chesapeake Bay; 300 fathoms.

208-Catapagurus sharreri. Deep-sea Hermit Crab. S. I. Off Martha's Vineyard, Mass.; 120 to 146 fathoms.

209-Munida sp.? S. I.

Off Martha's Vineyard, Mass.; 67 to 71 fathoms.

210-Homarus Americanus. American Lobster. S. I.

Vineyard Sound, Mass. 
211-C'ambarus aftinis. Cray-fish. S. I.

Potomac River.

212-Crangon vulgaris. Common Shrimp. S. I.

Off Cape Cod, Mass ; 26 fathoms.

213-Pandolus leptoceros. Deep-water prawn. S. I.

Off Martha's Vineyard, Mass.; 46 fathoms.

214-Palamonetes vulgaris. Common Prawn. S. I.

Narragansett Bay, R. I., shore.

215-Pagurus sp.? Hermit Crab.

Borneo.

216-Pagurus sp.? Hermit Crab.

217-Maja squinado. Large Sea-crab.

German Ocean.

218-IIomarus Americanus. Large specimen of the American Lobster.

New England coast.

219220 -Cambarus acutus. Cray-fish.

Grand Forks, Dakota.

\section{Class-Archnida.}

ORDER-AR'TIROGASTRA.

221-Scorpio. Scorpion.

ORDER-ARANEINA.

222-Tarantula lycosa. Tarantula Spider.

California.

22:3-Tarantula lycosa. Tarantula Spider. San Jose, California.

Presented by Mr. Goldwin S. Sprague, October, 's8. 


\section{Class-Myriapoda.}

224-Scolopendra perfida. Centipede.

225-Scolopendra sp.? Centipede.

Australia.

\section{SUB-KINGDOM-VERTEBRATA.}

Class-Leptocardii.

ORDER-PHARYNGOBRANCHII.

226-230-Amphioxus lanceolatus. Lancelet. Mediterranean, shore.

Presented by Henry Montgomery, University of North Dakota.

Class-Pisces (Fishes).

ORDER-MARSIPOBRANCHII.

231-Petromyzon sp.? Marine Lamprey.

$$
\text { ORDER-TELEOSTEI. }
$$

232--Morrhua vulgaris. Cod Fish. Skeleton of large specimen.

George's Banks.

233-Exocœtus sp.? Flying Fish.

Presented by Mr, M. Addison, Grand Forks, 1888. 


\section{Class-Amphibia.}

ORDER-URODELA.

234--Necturus lateralis (Menobranchus maculatus). Water Lizard or Mud Puppy. In alcohol. Animal 13 inches long. Captured November, 1888. Red River, Grand Forks, Dakota.

Presented by Henry Montgomery, November, 1888.

235--Necturus lateralis. Water Lizard. Mounted Skeleton.

236-Amphiuma tridactyla. Mounted skeleton.

South Carolina.

2:3-S-Salamandra maculata. Spotted Salamander. Mounted skeleton.

France.

2:3-2040-Amblystoma tigrinum. Tiger Salamander. In alcohol. Found alive six feet below the surface of the ground, near Grand Forks, in 1888, by Henry Montgomery, University of North Dakota.

241-246--Plethodon ervthronotus. Red Backed Salamander. Toronto, Ontario.

Presented by Henry Montgomery, University of North Dakota.

$$
\text { ORDER-ANOURA. }
$$

247-Rana mugiens. Bull Frog. Mounted skin.

248-Rana mugiens, Bull Frog. Mounted skeleton.

249-351-Rana balecina. Shad Frog. In alcohol.

Nelson County, Dakota.

252-254--Rana halecina. Shad Frog. In alcohol. 


\section{Class-Reptilia (Reptiles).}

ORDER - CHELONIA.

255-Chelopus guttatus. Turtle.

Massachusetts.

$$
\text { ORDER-LACERTILIA. }
$$

256-Phrynosoma cornutum. Ground Iguana or "Horned Toad." Mounted skin. California.

257-Acranthus viridis. Green Lizard. Mounted skin.

258-Draco volans. Flying Dragon. Mounted skin.

East Indies.

$$
\text { ORDER-OPHIDIA. }
$$

259-Crotalus durissus. Rattlesnake. Skeleton.

260-Eutæenia sirtalis. Garter Snake.

Grand Forks, Dakota.

$$
\text { ORDER-CROCODILIA. }
$$

261-Crocodilus palustris. Crocodile. Skull.

Ceylon.

\section{Class-Aves (Birds).}

Except otherwise stated, the following named specimens of birds are mounted skins.

$$
\text { ORDER - NATATORES (SWIMMERS). }
$$

262-Bernicla canadensis. Canada Wild Goose.

Pembina County, Dakota.

263 264--Chen hyperboreus, variety albatus. Lesser Snow Goose. North Dakota.

265-Olor (or Cy us) columbianus. Whistling Swan.

North Dakota. 
260-Mergus cucullatus. Hooded Merganser. Male. A handsome bird, an intermediate form between the ducks and geese. It nests in trees, and carries its young to water. Near Lakota, Nelson Co., Dakota.

Donated by Wm. Budge, Esq., Grand Forks, D. T', October 23, 1888 .

267-Mergus cucullatus. Hooded Merganser. Male.

Pembina County, Dakota.

268-Mergus serrator. Female of the Red-Breasted Merganser. 'The male_alone possesses a red breast.

Pembina County, Dakota.

269-Aix sponsa. Wood Duck or Summer Duck. This very pretty duck builds its nest in a tree, and with its beak carries its young to the water.

Pembina County, Dakota.

270 -Clangula albeola. Spirit Duck, Butter Ball, Buffle Head and Hell-diver are the vulgar names given to this duck with bright and iridescent plumage.

Pembina County, Dakota.

271-Anas boscas. Mallard. Female.

Pembina County, Dakota. Canvas-back duck. Female.

Pembina County, Dakota.

$273-$ Duck.

Pembina County, Dakota.

274-Spatula clypeata. Shoveller Duck or Broad Bill. Male. Pembina County, Dakota.

275-Querquedula discors. Blue-winged Teal. Female.

Pembina County, Dakota.

276--Mareea Americana. American Wigeon.

Fargo, Dakota:

277-Phalacrocorax carbo. Cormorant. Collected by Henry Montgomery, in 1887.

Devils Lake, Dakota. 
2is-Colymbus torquatus. Great Northern Loon or Diver. 'This bird has great diving powers, and is capable of swimming under the surface of the water for a distance of more than eighty rods.

Pembina County, Dakota.

ORDER-GRALLATORES (WADERS).

279 280-Grus canadensis. Northern Brown Crane or Sand Hill Crane. Large birds, $3 \frac{1}{2}$ feet high.

Pembina County, Dakota.

281-Fulica Americana. Mud Hen or Coot.

Pembina County, Dakota.

282 283-Porzana carolina. Carolina Rail or Small Water Rail.

North Dakota.

ORDER RASORES (SCRATCHERS).

284 28n-Cupidonia (upido. Pinnated Grouse or Prairie Hen. Pembina County. Dakota.

286 287-Pedicectes onlumbianus. Prairie Chicken of the Northwest. Sometimes called the Sharp-tailed Grouse.

North Dakota.

2s8-Bonasa umbella. Ruffled Grous" or "Partridge." This makes the well-known drumming noise of the "Partridge."

North Dakota.

ORDER-INSESSORES (PERCHERS).

289-291-Sturnella magna. Meadow Lark.

Pembina County, Dakota.

$292-$ Ground Robin.

Pembina County, Dakota.

293-Turdus migratorius. Migratory Thrush or American "Robin."

Pembina County, Dakota.

294-Icterus sp.? Oriole.

Pembina County, Dakota.

295 296-Icterus baltimore. Baltimore Oriole.

Pembina County, Dakota.

297 298-Ampelis garrulus. Bohemian Wax Wing.

Near Mandan, Dakota. 


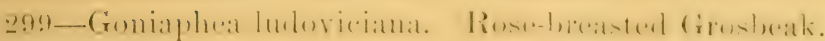

Pembina County, Dakota.

300301 -Quiscalus purpureus. Crow Blatk-bird.

Pembina County, Dakota.

302-Chrysomitris tristis. Thistle Bird or Gold Finch.

Pembina County, Dakota.

303-Tyranus earolinensis. King Bird or Bue-eater. This hird destroys multitudes of noxious insects.

Pembina Comnty, Dakota.

304--Ceryle alcyon. Belted Kingfisher.

Pembina County, Dakota. 305-Lanius borealis. Northeru Shrike or Butcher Bird.

Pembina County, Dakota.

306-Antrostomus vociferus. Night Jar or Whip-Poor-Will.

Pembina County, Dakota.

307 308--Corvus Americanus. Common Crow.

North Dakota.

309- Snow Bird. Pembina County, Dakota.

310- Stone Checker. Pembina County, Dakota. ORDER--SCANSORES (CLIMBERS).

311-Melanerpes erythrocephalus. Red-headed Woodpecker. Pembina County, Dakota. ORDER-RAPTORES (BIRDS OF PREY).

312-Nyctea seandiaca. Snowy Owl. Near Lakota, Nelson County, Dakota.

Presented by Wm. Budge, Esq., Frand Forks, Oct. 2:, 1888.

313-Nyctea scandiaca. Snowy Owl. East Grand Forks, Minn. Presented by Mr. Aldrich, East Grand Forks. 314 315-Jubo virchinianns. Great Horned Owl.

North Dakota.

$316-$

Red Sureceh Owl.

Pembina County, Dakota.

317-Rhinogryphus aura. 'Turkey Buzzard.

North Dikota.

318-Falco sparverius. Sparrow Hawk.

North Dakota. 
319-Buten swainsoni. American Buzzard, Swainson's Buzzard or Buzzard Hawk.

North Dakota.

320- Sharp-shinned Hawk.

Pembina County, Dakota.

321-Cathartes atrata. Black Vulture. Skeleton.

Southern States.

\title{
Class-Mammalia (Mammals).
}

\author{
ORDER--MARSUPIALIA.
}

322--Macropus giganteus. Giant Kangaroo. Skull.

Australia.

32:3-Marcropus rufus. Red Kangaroo. Mounted skin of large animal.

Victoria, Australia.

$$
\text { ORDER-EDENTATA. }
$$

324-Bradypus torquatus. Collared Sloth. Mounted Skin. Brazil, South America. ORDER-UNGULATA.

325-Equus caballus. Horse. Skeleton of left hind leg mounted.

326-Camelus Arabicus. Dromedary. Skull.

Arabia.

327--Alces Americanus. Moose. Pair of Antlers.

Near Lake of the Woods, Manitoba. 
32ミ-Ovis aries. Sheep. Mounted Skeleton.

329---Sus scrofigera. Wild Boar. Skull.

France.

ORDER-RODENTIA.

3:30-Spermophilus Franklini. Gray Gopher. Skull.

Grand Forks, Dakota.

:3:3-Spermophilus tridecemlineata. Striped Gopher. Skull.

Grand Forks, Dakota.

3:32-Geomys bursarius. Pocket Gopher. Skull.

Grand Forks, Dakota.

3:33-Cynomys ludovicianus. Prairie Dog. Skeleton.

North Nebraska.

3:34-Sciurus sciuropterus. Flying Squirrel. Mounted skin. Grand Forks, Dakota.

Presented by Prof. W. Merrifield, University of North Dakota.

335-Castor canadensis. Canada Beaver. Mounted skin.

Near Mandan, Dakota.

ORDER-CARNIVORA.

336-Taxidea Americana. American Badger. Mounted skin of adult female.

Pembina County, Dakota.

337-C'Taxidea Americana. American Badger. Mounted skin of young of the preceding.

Pembina County, Dakota.

338--Lynx canadensis. Canada Lynx or "Wild Cat." Killed January 4, 1889, in Walsh County, Dakota.

Presented by Dr. R. M. Evans, Minto, Dakota.

339-Felis domestica. Domestic Cat. Mounted skeleton.

340-Vulpes fulvus. American Fox or Red Fox. Mounted skin.

Mandan, Dakota.

341-Canis familiaris. Dog. Skull.

Presented by Mr. Louis O. Fiset, student of the

University of North Dakota, October, 18ss.

342-Canis latrans. Prairie Wolf or Coyote. Mounted skin. Near Ḿandan, Daknta. 
ORDER-INSECTIVORA.

343-Erinaceus Europæus. European Hedgehog. Mounted skeleton.

Germany.

ORDER- - CHEIROPTERA.

344-Pteropus polincephalus. Australian Fox Bat. Large, mounted skin.

New South Wales, Australia.

345-Pteropus Edwardsii. Edwards' Fox Bat. Mounted skeleton. Ceylon.

ORDER-QUADRUMANA.

346-Simia satyrus. Orang Outang. Skull.

Borneo.

\section{Discellaneous Gollection.}

1-Wine cup, made from Cypress wood grown upon the Mount of Olives, Palestine.

Presented by Mrs. C. L. Wording, Grand Forks, 1887. 2-Modern Indian Iron Hatchet.

Presented by Dr. R. M. Evans, Minto, Dakota, 1887.

3-Modern Indian Bead and Shell Ornaments.

Presented by Regent J. Twamley, 1886.

4-Horse Pistol, 21 inches long. Found at Indian modern burial scaffold, near Chadron, Nebraska.

Collected and presented by Henry Montgomery, 1885.

5-29-Twenty-five brass rings, each $2 \frac{1}{2}$ inches in diameter and open. Found with the aforesaid horse pistol, near Chadron, Nebraska.

Collected and presented by Henry Montgomery, 1885. 30 - Fencing Sword, made in Solingen. Found near the Inrian trail between Devils Like and the Turtle Mountains. Very old.

Presented by a resident of Devils Lake, Sept., 1887.

31-39-Arrow Heads. White quartz and dark colored chert. Fairfield, Conn.

Presented by Mr. Fred E. Silliman, 1888. 


\section{Devils Sake Collection.}

It is regretted that the study and arrangement of this large and important collection have not yet been completed, and that, therefore, but brief mention of it can be made in this edition of the catalogue.

This collection was made in the interest of the University

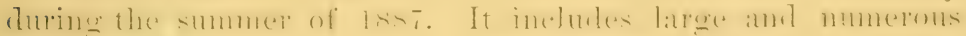
samples of the granite, syenite, limestone and other rocks in the

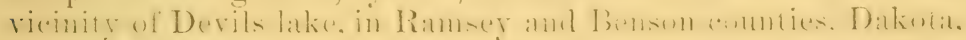
as well as skeletons, skulls, agate spear heads, agate chips, stone pipes, clay pipes, bone knives and skewers, many pearl, shell and bone ornaments, shell scoops or spoons, hieroglyphics on stone, pottery vessels, and copper and stone implements taken from numerous mounds in the same counties. These latter are believed to be the remains of an extinct race that ocempied Dakota many centuries ago.

There are about six hundred specimens in this collection. 




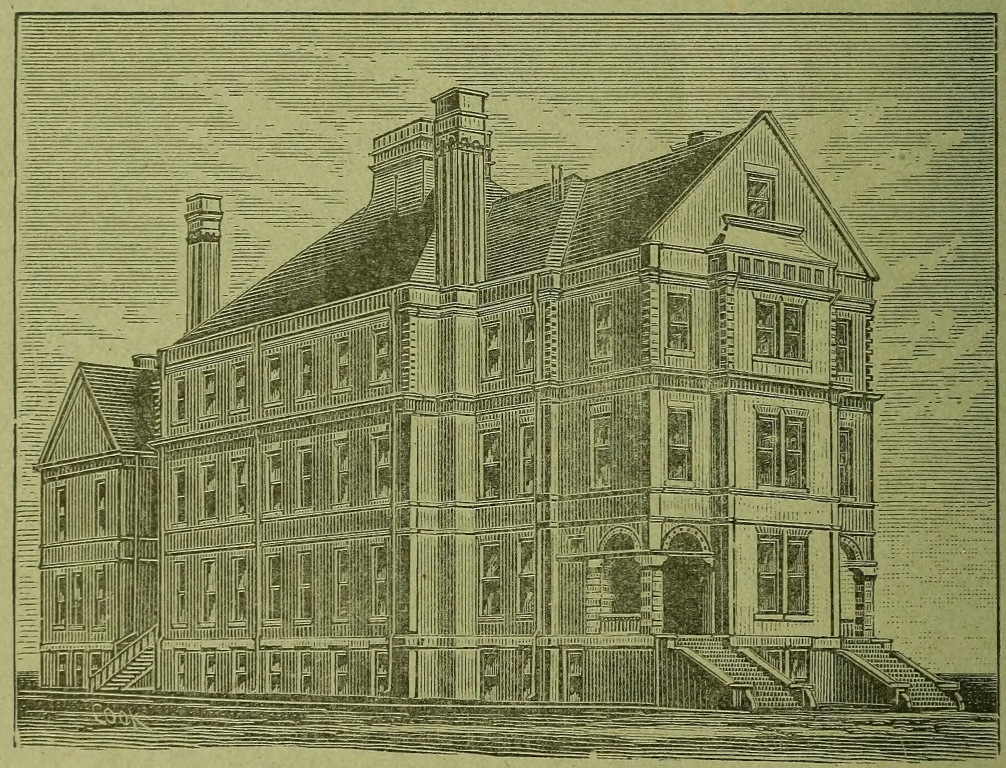


